\title{
What have worm models told us about the mechanisms of neuronal dysfunction in human neurodegenerative diseases? Dawn Teschendorf and Christopher D Link*
}

\author{
Address: Institute for Behavioral Genetics, University of Colorado, Boulder, CO, 80309, USA \\ Email: Dawn Teschendorf - dteschendorf@gmail.com; Christopher D Link* - linkc@colorado.edu \\ * Corresponding author
}

Published: 28 September 2009

Molecular Neurodegeneration 2009, 4:38 doi:10.1186/1750-1326-4-38
Received: I August 2009

Accepted: 28 September 2009

This article is available from: http://www.molecularneurodegeneration.com/content/4/I/38

(C) 2009 Teschendorf and Link; licensee BioMed Central Ltd.

This is an Open Access article distributed under the terms of the Creative Commons Attribution License (http://creativecommons.org/licenses/by/2.0), which permits unrestricted use, distribution, and reproduction in any medium, provided the original work is properly cited.

\begin{abstract}
The nematode worm Caenorhabditis elegans has become an intensely studied model organism, and worm studies have made significant contributions to developmental biology and other fields. The experimental advantages of $C$. elegans, particularly its simple anatomy, optical transparency, short lifespan, and facile genetics, have also led researchers to use this model to investigate neuronal cell degeneration and death. Worm studies of neurodegeneration can be divided into two general classes: studies in which mutations of $C$. elegans genes lead to neuronal dysfunction and death, and studies in which external manipulations (e.g., chemical treatments or introduction of engineered transgenes) are used to induce neurodegeneration. For both types of studies the primary approach has been to use forward genetic, reverse genetic, or candidate gene approaches to identify genes that modify neurodegeneration. The ease and relatively low cost of $C$. elegans propagation also suggests a role for these $C$. elegans models for compound screening. An excellent review has been previously published that summarizes much of the work done on mutationally-induced neuronal death in C. elegans [I]. This review focuses on studies that have attempted to model specific human neurodegenerative diseases using transgenic approaches. These studies have given us a variety of insights into the specific disruptions of cellular processes that may underlie human neurodegenerative diseases.
\end{abstract}

\section{Are worm models of human neurodegenerative diseases a good idea?}

Although invertebrate model systems (e.g., C. elegans or Drosophila) have significant experimental advantages, it is not self-evident that they are good approaches to study human neurodegeneration. As detailed below for C. elegans, there is strong support for the conservation of basic neuronal cellular functions between invertebrates and vertebrates. The critical question is therefore whether the specific neuronal cellular functions that are directly perturbed in neurodegenerative diseases are also conserved between these model systems and people. Given that the causal molecular/cellular insults underlying neurodegenerative conditions are not known (or at least controversial), the answer to this question is unclear. It is clear, though, that expression of specific human proteins linked to neurodegeneration (e.g., $\beta$-amyloid peptide, tau, $\alpha$ synuclein, etc.) leads to cellular toxicity in worms and flies. Minimally, invertebrate models should give us insight into (at least some) toxic activities of disease-relevant human proteins. The relevance of neurotoxic mechanisms established in worm or fly models necessarily need to be validated in mammalian systems. Thus, it is perhaps best to view worm models of neurodegeneration as 
"scout" systems that have the potential to generate important findings regarding mechanisms of neurotoxicity, with the caveat that all findings are probably provisional until extended to mammalian systems.

A major challenge in generating a worm neurodegeneration model is obtaining evidence that any observed pathology is specific and disease-relevant. One such piece of evidence for transgenic models is the demonstration that disease proteins containing mutations associated with familial forms of the disease are more toxic than wild type when expressed in worms [e.g., $\alpha$-synuclein containing familial Parkinson's disease (PD) substitutions should be more toxic than wild type $\alpha$-synuclein]. In addition, demonstrating that a disease model shows the same spectrum of neurotoxicty (e.g., preferential toxicity to dopaminergic neurons in PD models) and/or similar cell pathology (e.g., formation of Lewy body-like inclusions in PD models) as observed in the human disease also strengthens the case for the relevance of a model. It is reasonable to assume that worm models with cellular (or molecular) pathology more similar to the human disease have a stronger "presumption of relevance".

The strongest argument for developing worm models of neurodegeneration is that worms are amenable to unbiased genetic approaches, classically based on identifying forward mutations that modify the phenotype presented by a given model. In recent years this approach has been extended by using reverse genetic techniques such as RNA interference (RNAi). The value of these unbiased approaches lies in the two possible outcomes of a successful screen: either a gene previously linked to the disease is found, or a novel interactor is identified. In the first case the result strengthens previous findings, in the later case new insights may be gained. (Possibility \#2 is a corollary of a creed in the field: genetics is a way to answer questions you weren't smart enough to ask in the first place.) Of course, novel findings from worm models require extra skepticism and validation.

Additional advantages of worm models include the short lifecycle/lifespan and the ability to generate transgenic strains relatively rapidly. At $20^{\circ} \mathrm{C}$, the most common culture condition for $C$. elegans, the life cycle takes approximately 4 days and the mean lifespan is about 17 days. Thus, strains containing heritable (although typically unstable) transgenes can be constructed by microinjection in two weeks. (Derivation of completely stable, chromosomally integrated transgenes typically takes another month.) Combined with the ability to readily freeze and recover transgenic strains, it is feasible to generate large collections of strains expressing related transgenes [e.g., a series of engineered mutations in a protein or promoter region, a collection of Green Fluorescent Protein (GFP) reporters for a set of related genes, etc.]. This capacity allows comparative studies that would be very expensive and time-consuming to do in mice.

Although typically propagated on small agar-filled Petri plates, C. elegans can also be grown in microtiter wells, suggesting that worm models could be particularly appropriate for high-throughput drug screens.

\section{Relevant neuroanatomy of $C$. elegans}

Adult C. elegans hermaphrodites contain only 302 neurons, and the complete pattern of synaptic connections has been reconstructed by serial electron microscopy. Neuronal classes include chemosensory, mechanosensory, and thermosensory types; 75 motor neurons innervate the body wall muscles (excluding the head); 56 of these are cholinergic and 19 are GABAnergic. C. elegans larvae contain 4 serotonergic and 8 dopaminergic neurons. Formation, trafficking, and release of synaptic vesicles in C. elegans is highly conserved, employing many of the same proteins as are used in mammalian neurons. Because C. elegans is transparent throughout its life cycle, GFP fusions have been extensively used to visualize specific neurons and synapses in living animals. In some instances, neuronal death can be directly observed in living worms by the appearance of vacuolated neurons. More generally, GFP tagging of specific neurons allows observation of neuronal cell dystrophy or loss throughout the lifetime of the animal.

There are limitations to studying neurodegeneration in $C$. elegans. Worms do not have myelination or an active immune system, so they are presumably not appropriate for some neurodegenerative conditions such as multiple sclerosis. Practically, worm neurons are small and difficult to patch clamp, although recordings can be made from single identified neurons [2]. RNA interference (RNAi), a particularly useful tool in C. elegans, is often ineffective in neurons, necessitating the introduction of additional mutations to enhance neuronal RNAi efficacy.

\section{What can we expect to learn from worm models of neurodegeneration?}

Essentially all of the major age-associated neurodegenerative diseases (e.g., Alzheimer's, Parkinson's, ALS, etc.) have been linked to accumulation of specific proteins in the CNS. The neurodegenerative process in these diseases can be viewed as having three phases: accumulation of the toxic protein, toxic insult to neurons, and neuronal dysfunction and death. In theory, components in any of these phases can be identified by the identification of modifier genes in worm models of neurodegenerative diseases. This review will focus on a subset of $C$. elegans studies to illustrate how worm neurodegeneration models have been used to identify components of these phases of neurodegeneration. An inclusive list of studies employing 
transgenic C. elegans strains to study specific neurodegenerative diseases is shown in Table 1.

\section{Polyglutamine repeat diseases}

In 1999, Hart and colleagues described a C. elegans Huntington's disease (HD) model based on expression of a huntingtin fragment (exon 1) containing a 150 repeat polyglutamine (Ht-Q150) expansion [3]. Expression of this huntingtin fragment was driven by the osm-10 promoter, resulting in expression specifically in (non-essential) sensory neurons. Ht-Q150 expression was found to induce both neuronal dysfunction (demonstrated by the inability of chemosensory neurons to take up fluorescent dyes) and eventual death of chemosensory neurons (under conditions of osm-10::GFP co-expression). The apparent toxicity of Ht-Q150 was age-dependent but relatively mild, such that $13 \%$ of transgenic worms had dye uptake abnormalities at day 8 of development (midadulthood). This transgenic phenotype was subsequently used to identify mutations that enhanced Ht-Q150 toxicity, leading to the identification of pqe-1 [4]. Loss-of-function mutations in pqe-1 were found to dramatically enhance Ht-Q150-dependent chemosensory neuron death. pqe-1 encodes a nuclear protein rich in glutamines and prolines that also contains a conserved exonuclease domain (although PQE-1-homologous protein exist in mammals and flies, these proteins do not appear to contain glutamine/proline-rich domains). Given the cellular distributions of PQE-1 and Ht-Q150, these authors argued that wild type PQE-1 protein may protect from HtQ150 toxicity by competing for proteins sequestered by Ht-Q150.

The osm-10/Ht-Q150 model has also been used to investigate the roles of specific histone deacetylases in regulating huntingtin polyglutamine (Ht-polyQ) toxicity [5]. Studies in multiple models have demonstrated that expanded polyglutamine huntingtin can sequester CREB-binding

Table I: C. elegans transgenic models for human neurodegenerative diseases.

\begin{tabular}{|c|c|c|c|}
\hline Neurodegeneration-associated protein & Transgene expression & Promoter Sequence & References \\
\hline \multirow[t]{3}{*}{ Huntingtin::polyQ } & chemosensory neurons & osm- 10 & Faber et al [3] \\
\hline & mechanosensory neurons & mec-3 & Parker et al [II] \\
\hline & muscle & unc- 54 & Wang et al $[19]$ \\
\hline DRPLAP::polyQ & muscle & unc- 54 & Yamanaka et al [18] \\
\hline \multirow[t]{2}{*}{ GFP::polyQ } & muscle & unc-54 & Saytal et al [14] \\
\hline & pan-neuronal & rgef-I & Brignull et al [82] \\
\hline \multirow[t]{5}{*}{$\alpha$-synuclein } & pan-neuronal & aex-3 & Lakso et al [2I] \\
\hline & dopaminergic neurons & dat-I & \\
\hline & dopaminergic neurons & dat-I & Cao et al [22] \\
\hline & dopaminergic neurons & dat-I & Kuwahara et al [23] \\
\hline & pan-neuronal & unc-5 1 & Kuwahara et al [25] \\
\hline$\alpha$-synuclein::GFP & muscle & unc-54 & Hamamichi et al [26] \\
\hline$\alpha$-synuclein::YFP & muscle & unc- 54 & van Ham et al [27] \\
\hline \multirow{3}{*}{$\beta$-amyloid peptide } & muscle & unc-54 & Link [3I] \\
\hline & inducibe muscle & myo-3 & Link et al [38] \\
\hline & inducible pan-neuronal & snb-l & Wu et al [39] \\
\hline \multirow[t]{3}{*}{ tau } & pan-neuronal & $a e x-3$ & Kraemer et al [50] \\
\hline & mechanosensory neurons & mec-7 & Miyasaka et al [57] \\
\hline & pan-neuronal & rgef-I & Brandt et al [58] \\
\hline \multirow[t]{3}{*}{ SODI } & muscle & myo-3 & Oeda et al [60] \\
\hline & heat shock inducible & hsp-16.2 & \\
\hline & pan-neuronal & snb-l & Wang et al [6I] \\
\hline SODI::YFP & muscle & unc- 54 & Gidalevitz et al [62] \\
\hline LRRK2 & pan-neuronal & snb-l & Saha et al [63] \\
\hline mouse prion protein & muscle & unc- 54 & Park and Li [64] \\
\hline
\end{tabular}


protein (CBP) and its associated histone acetyltransferase activity $[6,7]$. Inhibition of histone deacetylase activity can counter this effect and subsequently reduce Ht-polyQ toxicity $[8,9]$. The ability to knock down specific gene expression in C. elegans by a simple feeding RNA interference procedure [10] allowed Bates et al to quickly assess the roles of 8 specific histone deacetylases in Ht-polyQ. Interestingly, knock down of one deacetylase (hda-3) reduced polyQ toxicity, while knockdown of the others increased toxicity as expected. Introduction of loss of function mutations for $h d a-1, h d a-4$, and sir-2.1 similarly enhanced Ht-polyQ toxicity.

A conceptually similar model for HD was developed by Parker et al [11] using the mec-3 promoter to express an Nterminal 57 residue fragment of huntingtin (with or without expanded polyglutamine repeats) fused to Green Fluorescent Protein (Ht-polyQ::GFP). The mec-3 promoter is active in 10 (non-essential) neurons, including the six touch receptor cells. Ht-polyQ::GFP toxicity was assayed by measuring touch responsivity in individual transgenic worms. As observed in the osm-10 model, increasing the number of polyglutamine repeats in the transgene led to increased deficits in touch sensitivity. Transgene-induced touch insensitivity was not associated with death of the touch cells, but could be associated with visible morphological abnormalities in touch cell axons. This model was subsequently used to demonstrate that over-expression of the sir-2.1 deacetylase could protect against HtpolyQ::GFP toxicity [12]. This result was extended by demonstrating that resveratrol, a demonstrated activator of sirtuin acetylases, could protect against Ht-polyQ toxicity in both worm and neuronal culture models. Interestingly, sir-2.1 or resveratrol protection in the C. elegans mec3/Ht-polyQ model was dependent on the FOXO transcription factor $d a f-16$, suggesting that this protection was not due to reversal of Ht-polyQ effects on histone acetylation per se. The mec-3/Ht-polyQ:: model has also been used to demonstrate a protective role for hipr-1, a homolog of the HIP1 huntingtin-interacting protein, against Ht-polyQ toxicity [13].

Perhaps a more general approach towards understanding polyglutamine repeat toxicity was initiated by the Morimoto lab, which examined the effects of short (Q19) and long (Q82) polyglutamine repeat lengths fused directly to GFP and expressed in C. elegans body wall muscle cells [14]. Expression of GFP::Q82 resulted in aggregate formation and induction of heat shock proteins. Subsequent studies with a series of YFP-polyQ fusions demonstrated a narrow threshold of polyQ repeat size (35-40) for induction of aggregation and toxicity [15]. Examination of transgenic worms expressing threshold-level glutamine repeats $(\mathrm{Q} 40)$ demonstrated a strong age dependence for aggregation as well as significant individual variability.
This model system was employed in an elegant series of studies to demonstrate that formation of polyQ aggregates generally disrupted protein homeostasis [16]. Introduction of an aggregation-prone YFP::polyQ transgene into a collection of temperature-sensitive missense mutations was found to dramatically enhance the phenotypes of these mutants, while conversely the presence of these missense mutations was found to enhance the aggregation of YFP::polyQ. These studies are consistent with a general "chaperone depletion" model that posits that aggregating protein toxicity results from competition for limited components of the protein homeostasis machinery. Indeed, a large scale RNAi screen for modifiers of YFP::polyQ aggregation identified a large set of genes involved in protein folding or degradation that increased polyQ aggregation when their expression was knocked down [17].

Additional C. elegans polyQ models have been generated with muscle-expressed polyglutamine repeats associated with either 17 amino acid residues derived from the dentatorubural pallidoluysian atrophy protein (DRPLAP) [18] or huntingtin exon 1 [19]. The former model has been used to demonstrate protective effects of $C$. elegans p97 homologs CDC-48.1 and CDC-48.2, while the latter model was used to show ubiquilin protection against polyQ toxicity. The muscle Ht-polyQ model has recently been used to implicate mitochondrial fission/fusion in huntingtin toxicity [20].

The studies described above illustrate the advantages (and disadvantages) of C. elegans neurodegeneration model systems, and the type of findings that can be made. The ability to undertake unbiased forward genetic screens enabled the identification of a novel gene involved in HtpolyQ toxicity ( $p q e-1)$. However, vertebrate homologs of pqe-1 do not contain the relevant glutamine/proline-rich portion of this protein, so the direct relevance to HD is unclear. The optical transparency and short lifespan of $C$. elegans readily allowed the demonstration of an agedependence for polyQ aggregation, something that is difficult to do directly in other model systems. Similarly, the existence of a large collection of characterized C. elegans mutations enabled the important demonstration of polyQ aggregation-dependent perturbation of protein homeostasis. The technically simple feeding RNAi protocol has enabled a number of candidate genes (e.g., hda-1, hda-4, sir-2.1, hipr-1, cdc-48.1,2 and dpr-1) to be implicated in in vivo polyQ toxicity. As is the case in other model systems, however, the use of different transgenic constructs by different research groups does confound making direct parallel between some studies. For example, it is unclear if toxicity in the neuronal expression osm$10 / \mathrm{Ht}-\mathrm{Q} 150$ model is due to the disruption of protein 
homeostasis, or if the huntingtin sequences themselves play an important role.

\section{Models of $\alpha$-synuclein toxicity}

$\alpha$-synuclein is a major component of Lewy bodies found in dystrophic dopaminergic neurons in Parkinson's disease (PD), and $\alpha$-synuclein mutations have been found to be casual in a relatively small number of familial PD cases. C. elegans models of synucleinopathy have been established with human $\alpha$-synuclein expression in either dopaminergic neurons [21-23] or pan-neuronally $[21,23]$. Lasko et al observed movement deficits in worms with pan-neuronal expression of wild type or A53T $\alpha$ synuclein, while Kuwahara did not observe any phenotypic effects of pan-neuronal wild type, A30P, or A53T $\alpha$ synuclein expression. (These differences may be a result of the specific promoters used in the transgene constructions; Lasko et al used the aex-3 promoter, while Kuwahara et al used the unc-51 promoter). However, all three groups have reported loss of either dopaminergic neuron cell bodies or dendrites when $\alpha$-synuclein expression is driven by the dopaminergic-specific dat-1 promoter. Kuwahara et al also observed preferential neuronal dysfunction in worms expressing mutant $\alpha$-synuclein (A53T or A30P) relative to wild type, measuring either dendritic loss or deficits in behaviors (slowing upon food sensation) known to be dopamine-dependent. The dat-1/wt $\alpha$ synuclein model of Cao et al was used to demonstrate the protective effects of the chaperone protein torsin A [22] and Rab1, a protein believed to function in Golgi vesicular trafficking [24]. The pan-neuronal $\alpha$-synuclein model of Kuwahara et al has been used in a large-scale feeding RNAi screen to identify enhancers of $\alpha$-synuclein toxicity, leading to the identification of a number of genes (e.g., apa-2, aps-2, eps-8 and rab-7) involved in the endocytic pathway [25]. Studies of C. elegans $\alpha$-synuclein models have thus strongly supported the link between $\alpha$-synuclein toxicity and intracellular (synaptic?) vesicle trafficking.

C. elegans models have also been constructed to look for genes specifically influencing $\alpha$-synuclein aggregation, following the approach initially developed for polyQinduced aggregation [17]. The Caldwell and Nollen groups both demonstrated that $\alpha$-synuclein::GFP (or YFP) fusion protein expressed in C. elegans muscle cells leads to the formation of visible fluorescent aggregates, as previously observed for GFP::polyQ $([26,27]$. Hamamichi et al initially tested 868 "hypothesis based" RNAi clones for enhancement of $\alpha$-syn::GFP aggregation. (This screen was sensitized by co-expression of TOR-2 to reduce initial aggregation levels.) Twenty genes were ultimately identified that increased $\alpha$-syn::GFP aggregation when their expression was knocked down early in development by feeding RNAi. Seven of these genes were tested for effects on $\alpha$-synuclein toxicity (not necessarily equivalent to aggregation) by transgenic overexpression in the dat-1/ $\alpha$ synuclein model; five were found to partially suppress dopaminergic neuron loss. Interestingly, these validated aggregation/toxicity enhancer genes included the vacuolar assembly/sorting protein vps-41 and the autophagyrelated atgr-7. van Ham et al similarly screened for genes that increased $\alpha$-syn::YFP aggregation after RNAi knockdown, in this case using the "whole genome" Ahringer feeding RNAi library, which contains $\sim 15,000$ clones ( $\sim 85 \%$ of the genome represented). 80 genes were identified, with an over-representation of lipid- and vesicleassociated genes. For three of these genes (sir-2.1, lagr-1, and ymel-1) suppression of $\alpha$-syn::YFP aggregation was independently confirmed using genetic loss-of-function mutations.

Perhaps the most interesting result from these two largescale screens is that essentially no overlap was identified between genes that suppressed GFP::polyQ and $\alpha$ syn::GFP aggregation. This observation is consistent with results from yeast studies and supports the view that aggregation of different disease-associated proteins is not equivalent. However, it should be pointed out that there is also no overlap in the genes identified in the Hamamichi et al and van Ham et al studies. This lack of overlap could be the result of technical differences between the two screens, and in fact non-congruence between similar C. elegans RNAi screens is not uncommon (e.g., $[28,29])$. Large scale RNAi screens in C. elegans appear to have an inherent variability, and thus negative results from these screens should be interpreted cautiously.

\section{Models of $\beta$ amyloid peptide toxicity}

The $\beta$-amyloid peptide $(\mathrm{A} \beta)$ is a primary component of senile plaques found in the brains of Alzheimer's disease (AD) patients, and the existence of mutations in the gene encoding A $\beta$ (Amyloid Precursor Protein, APP) in a subset of familial AD cases argues for a causal role for this peptide in this disease. $A \beta$ has also been found to accumulate in muscles of patients with Inclusion Body Myositis, a debilitating myopathy [30]. Initial attempts to use C. elegans to understand $\mathrm{A} \beta$ toxicity employed the unc-54 promoter to constitutively express a signal peptide::A $\beta$ minigene in body wall muscle [31]. Transgenicallyexpressed $\mathrm{A} \beta$ was found to accumulate intracellularly in muscle cells, and result in an age-dependent paralysis phenotype. This intracellular $A \beta$ was able to form amyloid dye-reactive deposits with a fibrillar ultrastructure (i.e., amyloid deposits) [32]. Single amino acid substitutions (e.g., Leu ${ }^{17}$ Pro) were identified that blocked amyloid formation in this model but did not reduce toxicity, suggesting that amyloid itself is not the toxic species [33]. The HSP-16 family of small heat-shock proteins was found to be induced by and co-immunoprecipitate with $\mathrm{A} \beta$ in this model [34], and ectopic expression of HSP-16 could sup- 
press $A \beta$ toxicity [35]. Cohen et al [36] used the unc-54/ signal peptide:: $\mathrm{A} \beta$ 1-42 model to investigate the roles of two important modulators of C. elegans lifespan, the insulin growth factor 1-like signaling (ILLS) pathway and heat shock factor (HSF), in A $\beta$ toxicity. RNAi knockdown of either daf-16 (the FOXO transcription factor that controls gene expression downstream of ILS) or $h s f-1$ enhanced $A \beta$ toxicity, while activation of DAF-16 (via RNAi knockdown of daf-2, a negative regulator of daf-16) suppressed toxicity. Bacterial deprivation, a form of dietary restriction that extends lifespan in C. elegans, has also been found to reduce $A \beta$ (and polyQ) toxicity in a HSF-dependent manner [37].

Overall, the results of studies with worms with constitutive muscle expression of either A $\beta$ or YFP::polyQ are similar, and suggest that in both cases the observed toxicity primarily results from a general perturbation of protein homeostasis (proteostasis). Interestingly, neither DAF-16 activation nor bacterial deprivation was observed to reduce the overall accumulation of $A \beta$, despite being protective. These results suggest that upregulation of the chaperone/protein folding machinery is protective either because this blocks the formation of some specific toxic form of $A \beta$ (supported by the results of Cohen et al, who found changes in the aggregation state of $A \beta$ correlating with toxicity) and/or because this compensates for a general depletion of chaperone capacity by deposition of $A \beta$ aggregates. It is an open question whether the unc-54/signal peptide::A $\beta$ 1-42 model is directly relevant to Alzheimer's disease, as the levels of intraneuronal $\mathrm{A} \beta$ accumulation in the brain are unlikely to reach the intracellular $A \beta$ levels generated in this model. However, results from this model may be generally instructive in that they suggest that the age-dependence of $\mathrm{AD}$ on other neurodegenerative conditions may stem from agedependent loss of the ability to maintain cellular protein homeostasis. It should be noted that this model may be more directly relevant to inclusion Body Myositis, given the parallels of intramuscular $A \beta$ accumulation.

Transgenic worms have also been engineered to inducibly express $\mathrm{A} \beta$ upon temperature upshift, either in muscle [38] or pan-neuronally [39]. This temperature inducibility was engineered by using transgene constructs with abnormally long 3' untranslated regions, resulting in transgenic transcripts that are subject to degradation by the mRNA surveillance system. Introduction of these transgenes into worms containing a temperature-sensitive mutation in a gene essential for mRNA surveillance (smg-1) resulted in strains that had a $\sim 5$-fold increase in transgenic transcripts when shifted from the permissive $\left(16^{\circ} \mathrm{C}\right)$ to non-permissive $\left(23^{\circ} \mathrm{C}\right)$ conditions [37]. An advantage of this system is that, at least for engineered muscle expression, transgenic strains have been constructed that have wild type movement at the permissive temperature but become rapidly ( $24 \mathrm{hr}$ ) and uniformly paralyzed upon temperature upshift. The reproducible phenotype of the myo-3/signal peptide::A $\beta$ 1-42/long 3' UTR model has allowed straightforward quantification of the effects of treatments on $A \beta$ toxicity, and has been used to demonstrate the protective effects of specific gingkolides [39]. This inducible model has also been used to demonstrate a role for autophagy in countering $A \beta$ toxicity [40]. Microarray studies with this model identified AIP-1, a conserved protein thought to be a positive regulator of proteasome function $[41,42]$, as an $\mathrm{A} \beta$-induced protein that protects against toxicity by reducing $A \beta$ accumulation [43]. Interestingly, a human homolog of AIP-1, AIRAPL, also reduces toxicity when coexpressed with $A \beta$.

Transgenic worms with pan-neuronal expression (signal peptide::A $\beta$ driven by the $s n b-1$ promoter) were found to have intraneuronal accumulation of $A \beta$ [44] but relatively mild phenotypes, including altered chemotaxis to benzaldehyde and hypersensitivity to exogenous serotonin [39]. It is unclear if the apparent differences in the severity of phenotypes resulting from muscle or neuronal expression reflect differences in the manner by which C. elegans muscle and neuronal cells respond to $A \beta$, or simply quantitative differences in the effective expression levels resulting from the use of different promoters. McColl et al [45] have recently demonstrated by mass spectrometry that in the unc-54/signal peptide::A $\beta$ 1-42 constitutive model the species of $A \beta$ that accumulates is actually $A \beta 3-42$, likely due to signal peptidase cleavage after $\mathrm{Ala}^{2}$ of the $\mathrm{A} \beta$ sequence. As the inducible models utilize the same signal peptide::A $\beta$ minigene, it is likely that all of these models produce the truncated $A \beta 3-42$. The 3-42 form of $A \beta$ is readily found in senile plaques, where the $\mathrm{N}$-terminal glutamate residue is often converted to pyroglutamate [46]. However, McColl and colleagues did not detect pyroglutamate-modified $\mathrm{A} \beta$ in the transgenic worm model.

\section{Tauopathy models}

Alzheimer's disease, as well as Pick's disease and some forms of frontotemporal lobar dementia (FTLD), are associated with intraneuronal accumulation of neurofibrillary tangles (NFTs) composed of the microtubule binding protein tau. Mutations in tau have been demonstrated to underlie familial FTLD linked to chromosome 17 (FTLD17) [47-49], causally linking tau to some forms of neurodegeneration. Kraemer et al established a C. elegans tauopathy model by expressing wild type or FTLD-17-mutant tau pan-neuronally using the aex-3 promoter [50]. This model system replicated key observations of the human disease: accumulation of insoluble, phosphorylated tau, evidence of age-dependent neuronal degeneration and loss, a clear organismal phenotype (uncoordinated move- 
ment), and greater toxicity in worms expressing FTLD-17mutant tau $\left(\mathrm{V}^{337} \mathrm{M}\right.$ and $\left.\mathrm{P}^{301} \mathrm{~L}\right)$ than in worms expressing wild type tau. The uncoordinated phenotype of the aex-3/ tau worms has been used for both classic forward genetic and RNAi-based reverse genetic screens, resulting in interesting (and perhaps unexpected) findings. Genetic screens based on chemical mutagenesis and positional cloning have identified two genes, sut-1 [51] and sut-2 [52], whose loss of function suppresses the tau-induced uncoordinated phenotype. sut-1 encodes a nematode-specific protein that binds Sm proteins and SmY, a small nematodespecific RNA of unknown function [53]. However, Kraemer and Schellenberg were able to use yeast two-hybrid and genetic studies to demonstrate that sut-1 interacts with UNC-34, a member of the conserved Ena/VASP protein family. They speculated that this interaction may modulate actin dynamics and thereby ultimately suppress tau pathology. sut-2 is a zinc finger-containing protein homologous to yeast Nab2 and human ZC3H14. Nab2 has demonstrated roles in the nuclear export of mRNA [54], and both Nab2 and ZC3H14 have been shown to bind polyadenosine sequences [55]. Guthrie et al also found by two yeast hybrid studies that C. elegans protein ZYG-12, and its human homolog HOOK2, can interact with SUT-2. Given that HOOK2 is a component of aggresomes, these authors suggested that sut-2 suppression of tau pathology could involve either aggresome effects on tau accumulation or, conversely, tau effects on proper aggresome formation.

The aex-3/tau V ${ }^{337} \mathrm{M}$ model was used as the basis for a full genome feeding RNAi screen $(16,757$ RNAi clones assayed), looking for modifiers of the tau-induced uncoordinated phenotype [56]. Although no suppressor RNAi clones were found, 60 genes were eventually identified that specifically enhanced the tau-induced phenotype when their expression was knocked down by RNAi. These genes encoded proteins with a surprising range of functions, including phosphorylation, chaperone activity, neurotransmission and signaling, RNA processing, and various enzymatic functions. Seven of these genes (sir-2.3, vap-1, lin-44, aex-1, acr-14, pxn-1, and mut-14) were independently tested by introducing genetic loss-of-function mutations into the aex-3/tau $\mathrm{V}^{337} \mathrm{M}$ background; in all cases the enhancement of the uncoordinated phenotype was recapitulated. The range of identified enhancer genes may reflect a multitude of steps by which neurons normally counter either toxic protein accumulation (e.g., altering tau modification, aggregation, or degradation) or its downstream consequences. Alternatively, the apparent interaction of these genes with tau pathology could result from a rather indirect synergism in which a reduction of gene function that normally results in a phenotypically undetectable compromise of neuronal function (e.g., reduction of $a c r-14$, one of the many acetylcholine recep- tors in C. elegans) nevertheless significantly exacerbates tau pathology in a perhaps additive fashion. In any case, the interacting genes identified by both forward and reverse genetic approaches with this model suggest potentially novel mechanisms of tau pathology that warrant investigation.

Tau pathology has also been engineered in C. elegans by expressing wild type and FTLD-17-mutant tau specifically in touch neurons, using the mec-7 promoter [57]. Transgenic expression of tau in the touch neurons resulted in an age-dependent loss of touch sensitivity, again with FTLD17-mutant tau ( $\mathrm{P}^{301} \mathrm{~L}$ and $\left.\mathrm{R}^{406} \mathrm{~W}\right)$ showing enhanced toxicity. This model was used to test the effects of HSP70 overexpression (mild suppression of mutant tau toxicity), GSK-3 overexpression (mild enhancement of tau toxicity), or genetic blockage of apoptosis (no effect on tau toxicity). Brandt et al [58] examined the role of phosphorylation in tau toxicity by using the rgef- 1 promoter to engineer pan-neuronal expression of wild type, pseudophosphorylated (10 specific kinase target serine residues changed to glutamate), or phosphorylationresistant (10 specific kinase target serine residues changed to alanine) tau. Both wild type and glutamate-substituted tau were observed to have similar age-dependent uncoordinated phenotypes, although worms expressing the glutamate-substituted tau had higher levels of abnormal motorneurons. (The alanine-substituted tau also induced an uncoordinated phenotype, although this was hard to interpret because transgenic lines expressing this modified tau all had significantly higher levels of tau expression.) Neuronal death was not observed in either the mec-7/tau or rgef-1/tau models. However, in both models neuronal outgrowth abnormalities were observed. While this could be indicative of developmental effects of tau expression on axon pathfinding, similar abnormal outgrowth has been observed as a result of regeneration of broken axons [59], suggesting the alternative possibility that tau expression results in fragile axonal processes more susceptible to movement-induced breakage.

\section{Other transgenic models}

Mutations in superoxide dismutase (SOD1) are the most common known cause of familial Amyotrophic Lateral Sclerosis (fALS). Oeda et al [60] generated the first C. elegans model of SOD1 toxicity by expressing wild type or fALS mutant SOD1 $\left(\mathrm{A}^{4} \mathrm{~V}, \mathrm{G}^{37} \mathrm{R}\right.$, or $\left.\mathrm{G}^{93} \mathrm{~A}\right)$, using either the muscle-specific myo-3 promoter or the heat-shock inducible hsp-16.2 promoter. While these transgenic worms were not reported to have discernable phenotypes under standard conditions, worms expressing the fALS mutant SOD1 were found to be preferentially sensitive to paraquat. Wang et al [61] engineered pan-neuronal SOD1 expression using the $s n b-1$ (synaptobrevin) promoter, expressing both wild type and G ${ }^{85} \mathrm{R}$ fALS mutant SOD1. Expression 
of SOD1 G85R (fused to YFP or unfused), but not wild type SOD1, resulted in a clear age-dependent inhibition of locomotion. Transgenic worms expressing $G^{85} R$ SOD1::YFP also had visible fluorescent aggregates in neurons, allowing a full genome feeding RNAi screen in a sensitized eri-1; lin-15b genetic background. As observed in the RNAi screens of the aex-3/tau model, both expected (e.g., chaperone-related genes such as $h s f-1$ ) and unexpected (topoisomerase gene top-1, TGF $\beta$ component $d b l$ 1 ) were recovered as aggregation/toxicity enhancers. (One aggregation/toxicity suppressor was also identified but not described in this study.) Transgenic worms expressing SOD1::YFP fusions have also been constructed by Gidalevitz et al [62], who used the unc-54 muscle-specific promoter to express wild type and $\mathrm{G}^{85} \mathrm{R}, \mathrm{G}^{93} \mathrm{~A}$, and truncated (127X) fALS mutant SOD1. As reported by Wang et al, fALS SOD1::YFP, but not wild type, formed visible aggregates. While transgenic worms expressing fALS mutant SOD $1:: Y F P$ had relatively mild phenotypes, introduction of temperature-sensitive missense mutations (the same mutant alleles that are enhanced by YFP::polyQ aggregation) strongly exacerbated SOD1::YFP toxicity in an fALS mutant-specific manner. This result nicely complements the previous YFP::polyQ study by the Morimoto group, and supports the idea that misfolded proteins (i.e., destabilized missense mutant proteins and fALS mutant SOD1) compete for limiting protein homeostasis machinery.

Mutations in leucine-rich repeat kinase 2 (LRRK2) are the most common known cause of familial Parkinson's disease (fPD). LRRK2 is highly conserved, and C. elegans contains a clear ortholog, lrk-1. Saha et al [63] have recently shown that knockdown of lrk-1 sensitizes worms to the mitochondrial toxic rotenone, while pan-neuronal expression of wild type human or G2019S fPD LRRK2 (driven by the $s n b-1$ promoter) protects against rotenone toxicity. However, transgenic LRRK2 expression also led to a preferential loss of dopaminergic neurons, with $\mathrm{G}^{2019} \mathrm{~S}$ fPD LRRK2 being measurably more toxic than wild type LRRK2. This model should be amenable to the approaches previously used in the transgenic $\alpha$-synuclein models.

Pathological forms of the prion protein (PrP) are believed to be the cause of fatal spongiform encephalies, including Creutzfeldt-Jakob disease (CJD), kuru, and Bovine Spongiform Encephaly (BSE). There is a single report describing the expression of mouse prion protein (residues 23-231) in C. elegans [64]. Muscle expression of a $\operatorname{MoPrP}(23-$ 231)::CFP fusion protein (but not the CFP-only control) was found to induce visible fluorescent aggregates and a variable "Dumpy" phenotype, associated with poor locomotion and sarcomere disruption. Parallel expression of PrP expressing the P102L mutation associated with Gerst-
mann-Straussler-Scheinker disease resulted in a more severe movement deficit, while co-expression of PrP containing a dominant negative mutation (MoPrP Q167R::YFP) with MoPrP::CFP reduced apparent toxicity. However, proteinase K-resistant MoPrP could not be detected in transgenic worms, suggesting that the infectious scrapie form of $\operatorname{PrP}$ (PrPsc) was not being formed. Given the lack of PrPsc formation and the fact that the transgenic constructs lack the $\mathrm{N}$-terminal lipidation sequences of PrP likely important in pathology, it is unclear if this model is directly relevant to infectious prion diseases or is instead more akin to the general protein aggregation toxicity models.

\section{So what have we learned from $C$. elegans transgenic models?}

While some of the findings from these models have been robustly demonstrated in other systems (e.g., the role of histone deacetylases in Ht::polyQ toxicity), some are clearly novel and/or have potentially broad significance to neurodegeneration. The studies of Gidalevitz et al [16] demonstrating how polyQ deposition alters the folding and function of other proteins provides the first clear experimental support for the hypothesis that aggregationinduced chaperone depletion (e.g., as suggested to explain the toxicity of ALS-mutant SOD1, [65]) can cause cellular dysfunction. The studies with worm $\alpha$-synuclein models have strongly implicated vesicular function in $\alpha$-synuclein toxicity [24-26]. Studies with treatments that modulate $C$. elegans lifespan have suggested why age is the primary risk factor for most neurodegenerative diseases [36,37]. The studies of Kraemer and colleagues in particular have identified a number of unexpected genes with a putative role in tau pathology $[51,52,56]$, explicitly demonstrating the advantages of $C$. elegans models that are amenable to unbiased experimental approaches.

\section{Future prospects}

New C. elegans neurodegeneration models are likely to be developed in the near future, taking advantage of both technical advantages (directed single copy transgene insertions, tetracycline-induced transgene expression), and the identification of new neurodegeneration-associated genes (e.g., TDP-43). Existing approaches have certainly not been exhausted, as genetic modifier screens have not been done to saturation, and some published large scale RNAi screens could still be expanded to fullgenome screens. Clearly, some of the C. elegans findings still await validation in mammalian models. This is particularly important with respect to unexpected modifier genes identified in RNAi screens, given the possibility that these genes could be an artifactual result of worm-specific biology or the highly engineered nature of the model system. An important question that has not been rigorously addressed is to what degree underlying pathological proc- 
esses overlap between the various neurodegeneration models. While some studies point to potential overlap of pathological processes (e.g., the common demonstration that loss of $h s f-1$ function enhances aggregation/toxicity), it remains to be determined if any suppressors in specific models also have effects in other neurodegeneration models (e.g., do sut-1 or sut-2 suppress $\alpha$-synuclein or polyQ toxicity?). Technical improvements in C. elegans handling (e.g., the COPAS worm sorter and microfluidic worm manipulation) will also likely enable more efficient use of transgenic worm models in compound screening.

\section{C. elegans neurodegeneration model check list}

A sufficient number of studies have now been undertaken using transgenic $C$. elegans neurodegeneration models that a set of considerations can be established as to what is needed to establish an informative model.

\section{I) Choice of expression system}

Expression of a human disease-associated protein involves the construction of a chimeric transgene employing a C. elegans promoter and a human cDNA. Promoters used are typically either pan-neuronal (e.g., unc-119, snb1 , aex-3, rgef-1, etc.) or targeted to specific neuronal subtypes (e.g., mec-7; mechanosensory neurons, osm-10; chemosensory neurons; dat-1; dopaminergic neurons, etc.). Body wall muscle-specific promoters (unc-54 and myo-3), which give strong and highly specific expression, have also been used. Promoter choice depends upon both technical considerations and specific questions being addressed. Many neurodegeneration-associated proteins are widely expressed (e.g., Abeta, tau, $\alpha$-synuclein), and thus pan-neuronal promoters may be logical choices for these types of proteins. An advantage to this approach is that if pan-neuronal expression leads to toxicity in specific neuronal sub-populations (e.g., pan-neuronal expression of $\alpha$-synuclein leading to specific dopaminergic neurotoxicity), it strengthens the validity of the model. However, if pan-neuronal expression of a disease-associated protein is strongly toxic, it may be impossible to recover transgenic strains in the first place. In these instances, targeting expression to non-essential neuronal sub-populations such as chemosensory neurons may be required.

Unfortunately, expression systems equivalent to the Gal4/ UAS or tetracycline-induction systems developed for Drosophila have not yet been described for C. elegans (although this will likely change in the near future, R. Baumeister, personal communication). Thus, cell- or tissuespecific transgene expression must be individually engineered, and conditional expression has been limited to engineering heat-shock-inducible transgenes (which can be confounded by the general effects of heat shock) or the use of a temperature-sensitive mRNA surveillance system that leads to temperature-dependent levels of transgene expression [38], but does not allow on/off regulation.

\section{2) Choice of expressed proteins}

Interpretation of the effects of heterologous protein expression is completely dependent upon parallel development of the appropriate transgenic controls. The preferable situation is to express in parallel wild type and familial disease mutant forms of a disease protein (e.g., as is available for tau, $\alpha$-synuclein, SOD1, etc). Enhanced toxicity of the disease mutant forms provides support for the relevance of the transgenic model. Alternatively, it may be possible to generate disease protein variants predicted to be non-toxic (e.g., making a kinase-dead version of a disease-associated protein kinase). Demonstrating that a single amino acid change in a disease protein renders it non-toxic strongly argues against "non-specific over-expression" interpretations of transgene toxicity. As many (if not all) neurodegeneration-associated proteins are aggregation-prone, it is also possible to control for general aggregation toxicity by parallel expression of a "generic" aggregating protein such as GFP::degron [66].

(It should be noted that comparison of transgenic animals expressing "more" and "less" toxic versions of diseaseassociated proteins is not routinely done with mouse transgenic models, as transgenic mice are typically compared to their non-transgenic siblings. However, given the relative ease of establishing transgenic C. elegans strains, there is no rationale for not generating parallel control transgenic strains.)

Transgenically expressed disease proteins can conceivably be expressed with epitope (e.g., FLAG or myc) or fluorescent protein tags, although this is often unnecessary and potentially risks altering transgenic protein activities. An advantage of expressing human genes in C. elegans is that there is generally little cross-reactivity between specific antibodies for the human protein and worm proteins, and therefore immunoblots and/or immunohistochemistry can readily be used to follow the expression of the transgenic protein if appropriate antibodies exist. Protein fusions to GFP or other fluorescent can be very useful for rapidly establishing transgene expression and for determining the sub-cellular distribution of the transgenic protein.

\section{3) Construction of transgenic worms}

There are currently three general approaches to establishing transgenic $C$. elegans strains. Transgenic strains with multicopy extrachromosomal arrays can readily be generated by gonad microinjection [67]. Irradiation and screening of these extrachromosomal transgenic strains can be used to subsequently derive completely stable, chromosomally integrated transgenic lines. Neither transgene copy number nor chromosomal insertion site can be controlled using this method. Transgenic strains can also be generated by ballistic transformation $[68,69]$, which can directly generate single- or low copy number- transgene 
insertions into the chromosome. The site of chromosomal insertion is also random in this case. The recently developed Mos1-mediated single copy insertion technique (MosSCI) employs transposon excision and repair to introduce single copy transgene insertions in specific chromosomal locations [70]. This approach has great potential to generate reproducible transgenic models that will simplify comparisons of independent transgenic strains (e.g., strains expressing different disease mutant forms of a neurodegeneration-associated protein) and avoid the complications associated with high-copy transgenic arrays (high expression, germline silencing, potential genetic instability, etc).

Construction of C. elegans transgenic strains generally requires co-introduction of a marker transgene that enables identification of transgenic worms independent of the (usually unknown) effects of the experimental disease-associated transgene. For transgenic strains generated by the muticopy array approach, the marker transgene can be easily co-introduced by adding marker transgene plasmid DNA to the injection mix, as all injected DNA is typically assembled into the transgenic array. A plasmid encoding dominant mutant collagen [rol-6(su1006)] was commonly used as a marker transgene in early transgenic constructs [67], as introduction of this marker leads to an easily recognized "Roller" phenotype that can be seen with a standard dissecting microscope. GFP-expressing constructs (e.g. myo-2 promoter::GFP) are also routinely used as marker transgenes. An alternative approach is to use a plasmid that encodes a transgene that can rescue a chromosomal mutant, resulting in transgenic worms with wild type phenotypes against a background of mutant non-transgenic worms. Plasmids encoding wild type LIN15 (rescuing a multivulval phenotype caused by recessive lin-15 mutations) or DPY-20 (rescuing a "Dumpy" phenotype caused by recessive $d p y-20$ mutations) have been used in this approach. Although this approach can simplify identification of transgenic worms, it does require using mutant backgrounds and has the potential confound of partial rescue of the chromosomal mutation complicating characterization of the experimental transgene phenotypes.

For ballistic- or MosSCI-mediated transformation, the marker transgene must be incorporated into the same plasmid as the experimental transgene. Expression of wild type UNC-119 protein has most commonly been used as the marker in these transformation techniques, with unc119 mutant worms as the recipient. This approach is advantageous not only because the transgenic rescue of the severely uncoordinated unc-119 phenotype is easily seen, but because this rescue can be selected for under appropriate conditions. unc-119 mutant worms cannot enter the starvation-resistant dauer larval stage, and thus starvation of populations of unc-119 mutant worms subjected to ballistic transformation strongly enriches for transformed worms.

\section{4) Demonstration of transgene expression}

The first step after establishment of a transgenic strain is demonstration of expression of the expected transgenic protein, typically done by immunoblot and/or immunohistochemistry. C. elegans adult worms contain $\sim 0.4 \mu \mathrm{g}$ of total protein, so 50-100 worms are sufficient material for one SDS-PAGE lane. Detection of transgenic proteins by immunoblot can be more difficult if expression is limited to small numbers of cells (e.g., the 8 dopaminergic neurons in C. elegans). Multiple protocols have been established for C. elegans whole mount immunohistochemistry [71], which can be used to establish transgene expression even if limited to a single cell in the worm. Transgenic proteins can of course be fused with GFP or other fluorescent proteins, which allows direct visualization of transgene expression at the risk of altering transgenic protein function.

\section{5) Establishment of transgene-associated phenotypes}

Genetic (or chemical) screens require phenotypes, and thus transgenic $C$. elegans models with weak or non-existent phenotypes are less likely to be informative. In some cases, transgenic expression of disease-associated proteins can lead to strong, easily discernable phenotypes, such as uncoordinated movement in worms with transgenic neuronal expression of tau [50] or paralysis in worms with muscle expression of $A \beta$ [31]. However, even weak transgenic phenotypes can be useful, as they can be effectively used for enhancer screens (e.g., [25]).

\section{Compound screening using $\mathbf{C}$. elegans models}

C. elegans neurodegeneration models have been used to test the effects of individual drugs (e.g., resveratrol in a polyQ model, [12]; acetaminophen in multiple PD models, [72]) or relatively small collections of compounds (e.g., subcomponents of Gingko biloba extracts in $A \beta$ toxicity models, [39], candidate Huntington's drugs in a polyQ model, [73]). Although large numbers of replicate C. elegans populations can be readily grown in small volumes (e.g., microtiter wells), a previous hindrance in moving from low- to high-throughput screenings (HTS) was the requirement for manual manipulation and phenotypic scoring of worms. Recent technical advances have now overcome many of the practical limitations in using C. elegans models for HTS, most prominently the development of the Complex Object Parametric Analyzer and Sorter (a.k.a. the COPAS "worm sorter", [74]). This instrument can rapidly sort individual worms on the basis of length, optical density, and fluorescence, and can be used to automate both manipulation and scoring. Burns et al [75] demonstrated how this technology could be incorpo- 
rated with a high-throughput digital imager and data management software for precisely scoring phenotype. This protocol was used by Kwok et al [76] to effectively screen four libraries comprised of 14,100 small molecules, which resulted in their discovery of a new L-type calcium channel antagonist. The COPAS instrument has recently been used for high-throughput neurotoxin screening [77], suggesting that this instrument should be equally applicable for screening for neuroprotective compounds in various neurodegeneration models.

For some relevant phenotypes, such as axonal regeneration after microsurgery [78] or maintenance of motorneuron synapses, subcellular observations are required. Techniques are currently being developed that may allow these challenging measurements to be automated, thereby further expanding HTS possibilities. In 2007 the Yanik group at MIT introduced a series of microfluidic devices, otherwise known as lab-on-chip technology, applicable for manipulating C. elegans [79]. Microfluidic devices maneuver fluids and specimens on a sub-millimeter scale via the use of layered control channels and valves. Given the size of a C. elegans adult $(\sim 1 \mathrm{mM}$ in length and $0.1 \mathrm{mM}$ in diameter), and that they are able to live out their 2-3 week lifespan suspended in liquid media, worms are an ideal animal model for this technology. These microfluidic devices can be configured with 3-dimensional imaging that allows for subcellular resolution. Additional key components of these devices are the manner with which immobilization of worms is achieved as well as the capability of continuous recirculation. A worm is captured by way of a suction channel, which restricts its movement and positions the worm in a linear fashion providing for optimal imaging. Based upon its phenotype, the animal is then either discarded or collected in a separate chamber through a series to flow valves controls. In 2008, the Hang Lu group demonstrated that integrating this lab-on-chip technology and automated multiparametric analysis could effectively identify and sort worms according to cellular and subcellular phenotypes with 95\% accuracy [80]. This technology has also facilitated procedures such as those used in the in vivo ultrafast laser nanosurgery study conducted by Ben-Yakar and Bourgeois [81]. By integrating lab-on-chip technology and femtosecond laser nanosurgery they were able to increase the level of precision and speed with which cellular and subcellular laser ablations can be performed, suggesting the feasibility of largescale in vivo neural degeneration and regeneration studies.

Despite the technical advances relative to HTS in C. elegans, biological considerations may still be the most important factor in the potential usefulness of C. elegans neurodegeneration models for drug identification. The $C$. elegans cuticle is impermeant to many compounds that readily diffuse into tissue culture cells, and thus com- pound entry may require ingestion along with the standard E. coli food source. For a given compound, there is currently no way to predict what final tissue concentration will result from a given external compound dose, so dose/ responses need to be tested empirically. It can be argued that the major advantage of compound screening in C. elegans is also the major disadvantage: protective compounds can be identified without any assumptions about neurotoxic mechanisms, however, when protective compounds are identified, the molecular mechanism of protection may be unknown. This ignorance can be a major hurdle in further drug development, as it limits both compound optimization and predictions about possible side effects. Nevertheless, investigations of protective compounds can be a powerful approach to ultimately understanding the molecular mechanisms of neurodegeneration.

\section{Competing interests}

The authors declare that they have no competing interests.

\section{Authors' contributions}

DT and CDL both contributed to the writing of this manuscript and approve of the final submitted text.

\section{Acknowledgements}

The authors would like to thank Gin Fonte for the careful reading of this manuscript. CDL is supported by the NIH (grants ROI AGO I 2423 and ROI NS063964) and a Zenith Award from the Alzheimer's Association.

\section{References}

I. Kourtis N, Tavernarakis N: Non-developmentally programmed cell death in Caenorhabditis elegans. Semin Cancer Biol 2007, 17:122-33.

2. Ramot D, Maclnnis BL, Goodman MB: Bidirectional temperaturesensing by a single thermosensory neuron in $C$. elegans. Nat Neurosci 2008, I I:908-15.

3. Faber PW, Alter JR, MacDonald ME, Hart AC: Polyglutaminemediated dysfunction and apoptotic death of a Caenorhabditis elegans sensory neuron. Proc Natl Acad Sci USA 1999, 96: 179-84.

4. Faber PW, Voisine C, King DC, Bates EA, Hart AC: Glutamine/proline-rich PQE-I proteins protect Caenorhabditis elegans neurons from huntingtin polyglutamine neurotoxicity. Proc Natl Acad Sci USA 2002, 99: $17131-6$.

5. Bates EA, Victor M, Jones AK, Shi Y, Hart AC: Differential contributions of Caenorhabditis elegans histone deacetylases to huntingtin polyglutamine toxicity. J Neurosci 2006, 26:2830-8.

6. McCampbell A, Taylor JP, Taye AA, Robitschek J, Li M, Walcott J, Merry D, Chai Y, Paulson H, Sobue G, Fischbeck KH: CREB-binding protein sequestration by expanded polyglutamine. Hum Mol Genet 2000, 9:21 97-202.

7. Jiang H, Nucifora FC Jr, Ross CA, DeFranco DB: Cell death triggered by polyglutamine-expanded huntingtin in a neuronal cell line is associated with degradation of CREB-binding protein. Hum Mol Genet 2003, I 2: I- 12.

8. Steffan JS, Bodai L, Pallos J, Poelman M, McCampbell A, Apostol BL, Kazantsev A, Schmidt E, Zhu YZ, Greenwald M, Kurokawa R, Housman DE, Jackson GR, Marsh JL, Thompson LM: Histone deacetylase inhibitors arrest polyglutamine-dependent neurodegeneration in Drosophila. Nature 200I, 4I3:739-43.

9. McCampbell A, Taye AA, Whitty L, Penney E, Steffan JS, Fischbeck $\mathrm{KH}$ : Histone deacetylase inhibitors reduce polyglutamine toxicity. Proc Natl Acad Sci USA 200I, 98:15 I79-84. 
10. Timmons L, Fire A: Specific interference by ingested dsRNA. Nature 1998, 395:854.

II. Parker JA, Connolly JB, Wellington C, Hayden M, Dausset J, Neri C: Expanded polyglutamines in Caenorhabditis elegans cause axonal abnormalities and severe dysfunction of PLM mechanosensory neurons without cell death. Proc Natl Acad Sci USA 200I, 98:133|8-23.

12. Parker JA, Arango M, Abderrahmane S, Lambert E, Tourette C, Catoire $\mathrm{H}$, Neri C: Resveratrol rescues mutant polyglutamine cytotoxicity in nematode and mammalian neurons. Nat Genet 2005, 37:349-50.

13. Parker JA, Metzler M, Georgiou J, Mage M, Roder JC, Rose AM, Hayden MR, Neri C: Huntingtin-interacting protein I influences worm and mouse presynaptic function and protects Caenorhabditis elegans neurons against mutant polyglutamine toxicity. J Neurosci 2007, 27: I I056-64.

14. Satyal SH, Schmidt E, Kitagawa K, Sondheimer N, Lindquist S, Kramer JM, Morimoto RI: Polyglutamine aggregates alter protein folding homeostasis in Caenorhabditis elegans. Proc Natl Acad Sci USA 2000, 97:5750-5.

I5. Morley JF, Brignull HR, Weyers JJ, Morimoto RI: The threshold for polyglutamine-expansion protein aggregation and cellular toxicity is dynamic and influenced by aging in Caenorhabditis elegans. Proc Natl Acad Sci USA 2002, 99: 10417-22.

16. Gidalevitz T, Ben-Zvi A, Ho KH, Brignull HR, Morimoto RI: Progressive disruption of cellular protein folding in models of polyglutamine diseases. Science 2006, 3 I I: | 47 I-4.

17. Nollen EA, Garcia SM, van Haaften G, Kim S, Chavez A, Morimoto RI, Plasterk RH: Genome-wide RNA interference screen identifies previously undescribed regulators of polyglutamine aggregation. Proc Natl Acad Sci USA 2004, 10 1:6403-8.

18. Yamanaka K, Okubo Y, Suzaki T, Ogura T: Analysis of the two p97I VCP/Cdc48p proteins of Caenorhabditis elegans and their suppression of polyglutamine-induced protein aggregation. J Struct Biol 2004, 146:242-50.

19. Wang H, Lim PJ, Yin C, Rieckher M, Vogel BE, Monteiro MJ: Suppression of polyglutamine-induced toxicity in cell and animal models of Huntington's disease by ubiquilin. Hum Mol Genet 2006, I 5: 1025-4I.

20. Wang H, Lim PJ, Karbowski M, Monteiro MJ: Effects of overexpression of huntingtin proteins on mitochondrial integrity. Hum Mol Genet 2009, I 8:737-52.

21. Lakso M, Vartiainen S, Moilanen AM, Sirvio J, Thomas JH, Nass R, Blakely RD, Wong G: Dopaminergic neuronal loss and motor deficits in Caenorhabditis elegans overexpressing human alpha-synuclein. J Neurochem 2003, 86: 165-72.

22. Cao S, Gelwix CC, Caldwell KA, Caldwell GA: Torsin-mediated protection from cellular stress in the dopaminergic neurons of Caenorhabditis elegans. J Neurosci 2005, 25:380I-I2.

23. Kuwahara T, Koyama A, Gengyo-Ando K, Masuda M, Kowa H, Tsunoda M, Mitani S, Iwatsubo T: Familial Parkinson mutant alphasynuclein causes dopamine neuron dysfunction in transgenic Caenorhabditis elegans. J Biol Chem 2006, 28 I:334-40.

24. Cooper AA, Gitler AD, Cashikar A, Haynes CM, Hill KJ, Bhullar B, Liu K, Xu K, Strathearn KE, Liu F, Cao S, Caldwell KA, Caldwell GA, Marsischky G, Kolodner RD, Labaer J, Rochet JC, Bonini NM, Lindquist S: Alpha-synuclein blocks ER-Golgi traffic and RabI rescues neuron loss in Parkinson's models. Science 2006, 3 I 3:324-8.

25. Kuwahara T, Koyama A, Koyama S, Yoshina S, Ren CH, Kato T, Mitani S, Iwatsubo T: A systematic RNAi screen reveals involvement of endocytic pathway in neuronal dysfunction in alpha-synuclein transgenic C. elegans. Hum Mol Genet 2008, I 7:2997-3009.

26. Hamamichi S, Rivas RN, Knight AL, Cao S, Caldwell KA, Caldwell GA: Hypothesis-based RNAi screening identifies neuroprotective genes in a Parkinson's disease model. Proc Natl Acad Sci USA 2008, 105:728-33.

27. van Ham TJ, Thijssen KL, Breitling R, Hofstra RM, Plasterk RH, Nollen EA: C. elegans model identifies genetic modifiers of alphasynuclein inclusion formation during aging. PLOS Genet 2008, 4(3): 1000027.

28. Dillin A, Hsu AL, Arantes-Oliveira N, Lehrer-Graiwer J, Hsin H, Fraser AG, Kamath RS, Ahringer J, Kenyon C: Rates of behavior and aging specified by mitochondrial function during development. Science 2002, 298:2398-40I.
29. Lee SS, Lee RY, Fraser AG, Kamath RS, Ahringer J, Ruvkun G: A systematic RNAi screen identifies a critical role for mitochondria in C. elegans longevity. Nat Genet 2003, 33:40-8.

30. Vattemi G, Nogalska A, King Engel W, D'Agostino C, Checler F, Askanas $V$ : Amyloid-beta42 is preferentially accumulated in muscle fibers of patients with sporadic inclusion-body myositis. Acta Neuropathol 2009, I I 7:569-74.

31. Link CD: Expression of human beta-amyloid peptide in transgenic Caenorhabditis elegans. Proc Natl Acad Sci USA 1995, 92:9368-72.

32. Link CD, Johnson CJ, Fonte V, Paupard M, Hall DH, Styren S, Mathis CA, Klunk WE: Visualization of fibrillar amyloid deposits in living, transgenic Caenorhabditis elegans animals using the sensitive amyloid dye, X-34. Neurobiol Aging 200I, 22:2 I7-26.

33. Fay DS, Fluet $A$, Johnson $C$ J, Link $C D$ : In vivo aggregation of betaamyloid peptide variants. J Neurochem 1998, 71:1616-25.

34. Fonte V, Kapulkin V, Taft A, Fluet A, Friedman D, Link CD: Interaction of intracellular beta amyloid peptide with chaperone proteins. Proc Natl Acad Sci USA 2002, 99:9439-44.

35. Fonte V, Kipp DR, Yerg J 3rd, Merin D, Forrestal M, Wagner E, Roberts CM, Link CD: Suppression of in vivo beta-amyloid peptide toxicity by overexpression of the HSP-16.2 small chaperone protein. J Biol Chem 2008, 283:784-9I.

36. Cohen E, Bieschke J, Perciavalle RM, Kelly JW, Dillin A: Opposing activities protect against age-onset proteotoxicity. Science 2006, 31 3:1604-10.

37. Steinkraus KA, Smith ED, Davis C, Carr D, Pendergrass WR, Sutphin GL, Kennedy BK, Kaeberlein M: Dietary restriction suppresses proteotoxicity and enhances longevity by an hsf-I-dependent mechanism in Caenorhabditis elegans. Aging Cell 2008, 7:394-404.

38. Link CD, Taft A, Kapulkin V, Duke K, Kim S, Fei Q, Wood DE, Sahagan BG: Gene expression analysis in a transgenic Caenorhabditis elegans Alzheimer's disease model. Neurobiol Aging 2003, 24:397-4I3

39. Wu Y, Wu Z, Butko P, Christen Y, Lambert MP, Klein WL, Link CD, Luo Y: Amyloid-beta-induced pathological behaviors are suppressed by Ginkgo biloba extract EGb $76 \mathrm{I}$ and ginkgolides in transgenic Caenorhabditis elegans. J Neurosci 2006, 26: I 3 I 02-13.

40. Florez-McClure ML, Hohsfield LA, Fonte G, Bealor MT, Link CD: Decreased insulin-receptor signaling promotes the autophagic degradation of beta-amyloid peptide in C. elegans. Autophagy 2007, 3:569-80.

41. Stanhill A, Haynes CM, Zhang Y, Min G, Steele MC, Kalinina J, Martinez E, Pickart CM, Kong XP, Ron D: An arsenite-inducible I9S regulatory particle-associated protein adapts proteasomes to proteotoxicity. Mol Cell 2006, 23:875-85.

42. Yun C, Stanhill A, Yang Y, Zhang Y, Haynes CM, Xu CF, Neubert TA, Mor A, Philips MR, Ron D: Proteasomal adaptation to environmental stress links resistance to proteotoxicity with longevity in Caenorhabditis elegans. Proc Natl Acad Sci USA 2008, 105:7094-9.

43. Hassan WM, Merin DA, Fonte V, Link CD: AIP-I ameliorates beta-amyloid peptide toxicity in a Caenorhabditis elegans Alzheimer's disease model. Hum Mol Genet 2009, 18:2739-47.

44. Link $C D$ : $C$. elegans models of age-associated neurodegenerative diseases: lessons from transgenic worm models of Alzheimer's disease. Exp Gerontol 2006, 4I:1007-13.

45. McColl G, Roberts BR, Gunn AP, Perez KA, Tew DJ, Masters CL, Barnham KJ, Cherny RA, Bush Al: The Caernorhabditis elegans Abetal-42 model of Alzheimer's disease predominantly expresses Abeta3-42. J Biol Chem 2009, 284(34):22697-702.

46. Saido TC, Iwatsubo T, Mann DM, Shimada H, Ihara Y, Kawashima S: Dominant and differential deposition of distinct beta-amyloid peptide species, A beta N3(pE), in senile plaques. Neuron 1995, I 4:457-66.

47. Hutton M, Lendon CL, Rizzu P, Baker M, Froelich S, Houlden H, Pickering-Brown S, Chakraverty S, Isaacs A, Grover A, Hackett J, Adamson J, Lincoln S, Dickson D, Davies P, Petersen RC, Stevens M, de Graaff E, Wauters E, van Baren J, Hillebrand M, Joosse M, Kwon JM, Nowotny P, Che LK, Norton J, Morris JC, Reed LA, Trojanowski J, Basun H, Lannfelt L, Neystat M, Fahn S, Dark F, Tannenberg T, Dodd PR, Hayward N, Kwok JB, Schofield PR, Andreadis A, Snowden J, Craufurd D, Neary D, Owen F, Oostra BA, Hardy J, Goate A, van Swieten J, Mann D, Lynch T, Heutink P: Association of missense 
and 5 '-splice-site mutations in tau with the inherited dementia FTDP-17. Nature 1998, 393:702-5.

48. Poorkaj P, Bird TD, Wijsman E, Nemens E, Garruto RM, Anderson L, Andreadis A, Wiederholt WC, Raskind M, Schellenberg GD: Tau is a candidate gene for chromosome 17 frontotemporal dementia. Ann Neurol 1998, 43:815-25.

49. Spillantini MG, Murrell JR, Goedert M, Farlow MR, Klug A, Ghetti B: Mutation in the tau gene in familial multiple system tauopathy with presenile dementia. Proc Natl Acad Sci USA 1998, 95:7737-4I.

50. Kraemer BC, Zhang B, Leverenz JB, Thomas JH, Trojanowski JQ, Schellenberg GD: Neurodegeneration and defective neurotransmission in a Caenorhabditis elegans model of tauopathy. Proc Natl Acad Sci USA 2003, 100:9980-5.

5I. Kraemer BC, Schellenberg GD: SUT-I enables tau-induced neurotoxicity in C. elegans. Hum Mol Genet 2007, 16:1959-71.

52. Guthrie CR, Schellenberg GD, Kraemer BC: SUT-2 potentiates tau-induced neurotoxicity in Caenorhabditis elegans. Hum Mol Genet 2009, 18:1825-38.

53. MacMorris M, Kumar M, Lasda E, Larsen A, Kraemer B, Blumenthal T: A novel family of $C$. elegans snRNPs contains proteins associated with trans-splicing. Rna 2007, I 3:5 II-20.

54. Suntharalingam M, Alcazar-Roman AR, Wente SR: Nuclear export of the yeast mRNA-binding protein $\mathrm{Nab2}$ is linked to a direct interaction with Gfd I and to Gle I function. J Biol Chem 2004, 279:35384-91.

55. Kelly SM, Pabit SA, Kitchen CM, Guo P, Marfatia KA, Murphy TJ, Corbett $\mathrm{AH}$, Berland KM: Recognition of polyadenosine RNA by zinc finger proteins. Proc Natl Acad Sci USA 2007, I 04: I 2306-I I.

56. Kraemer BC, Burgess JK, Chen JH, Thomas JH, Schellenberg GD: Molecular pathways that influence human tau-induced pathology in Caenorhabditis elegans. Hum Mol Genet 2006, 15:1483-96.

57. Miyasaka T, Ding Z, Gengyo-Ando K, Oue M, Yamaguchi H, Mitani S, Ihara $Y$ : Progressive neurodegeneration in $C$. elegans model of tauopathy. Neurobiol Dis 2005, 20:372-83.

58. Brandt R, Gergou A, Wacker I, Fath T, Hutter H: A Caenorhabditis elegans model of tau hyperphosphorylation: induction of developmental defects by transgenic overexpression of Alzheimer's disease-like modified tau. Neurobiol Aging 2009, 30:22-33.

59. Hammarlund M, Jorgensen EM, Bastiani MJ: Axons break in animals lacking beta-spectrin. J Cell Biol 2007, I 76:269-75.

60. Oeda T, Shimohama S, Kitagawa N, Kohno R, Imura T, Shibasaki H, Ishii $\mathrm{N}$ : Oxidative stress causes abnormal accumulation of familial amyotrophic lateral sclerosis-related mutant SOD I in transgenic Caenorhabditis elegans. Hum Mol Genet 200I, 10:2013-23.

61. Wang J, Farr GW, Hall DH, Li F, Furtak K, Dreier L, Horwich AL: An ALS-linked mutant SODI produces a locomotor defect associated with aggregation and synaptic dysfunction when expressed in neurons of Caenorhabditis elegans. PLoS Genet 2009, 5(I):el000350.

62. Gidalevitz T, Krupinski T, Garcia S, Morimoto RI: Destabilizing protein polymorphisms in the genetic background direct phenotypic expression of mutant SODI toxicity. PLoS Genet 2009, 5(3):e 1000399.

63. Saha S, Guillily MD, Ferree A, Lanceta J, Chan D, Ghosh J, Hsu CH, Segal L, Raghavan K, Matsumoto K, Hisamoto N, Kuwahara T, Iwatsubo T, Moore L, Goldstein L, Cookson M, Wolozin B: LRRK2 modulates vulnerability to mitochondrial dysfunction in Caenorhabditis elegans. J Neurosci 2009, 29:9210-8.

64. Park KW, Li L: Cytoplasmic expression of mouse prion protein causes severe toxicity in Caenorhabditis elegans. Biochem Biophys Res Commun 2008, 372:697-702.

65. Bruening W, Roy J, Giasson B, Figlewicz DA, Mushynski WE, Durham HD: Up-regulation of protein chaperones preserves viability of cells expressing toxic $\mathrm{Cu} / \mathrm{Zn}$-superoxide dismutase mutants associated with amyotrophic lateral sclerosis. J Neurochem 1999, 72:693-9.

66. Link CD, Fonte V, Hiester B, Yerg J, Ferguson J, Csontos S, Silverman $\mathrm{MA}$, Stein $\mathrm{GH}$ : Conversion of green fluorescent protein into a toxic, aggregation-prone protein by $\mathrm{C}$-terminal addition of a short peptide. J Biol Chem 2006, 28I:1808-16.

67. Mello CC, Kramer JM, Stinchcomb D, Ambros V: Efficient gene transfer in $C$. elegans: extrachromosomal maintenance and integration of transforming sequences. Embo J |991, 10:3959-70.

68. Wilm T, Demel P, Koop HU, Schnabel H, Schnabel R: Ballistic transformation of Caenorhabditis elegans. Gene 1999, 229:3I-5.

69. Jackstadt P, Wilm TP, Zahner H, Hobom G: Transformation of nematodes via ballistic DNA transfer. Mol Biochem Parasitol 1999, 103:26I-6.

70. Frokjaer-Jensen C, Davis MW, Hopkins CE, Newman BJ, Thummel JM, Olesen SP, Grunnet M, Jorgensen EM: Single-copy insertion of transgenes in Caenorhabditis elegans. Nat Genet 2008, 40:1375-83.

7I. Duerr JS: Immunohistochemistry. WormBook 2006: I-6I.

72. Locke CJ, Fox SA, Caldwell GA, Caldwell KA: Acetaminophen attenuates dopamine neuron degeneration in animal models of Parkinson's disease. Neurosci Lett 2008, 439:I29-33.

73. Voisine C, Varma H, Walker N, Bates EA, Stockwell BR, Hart AC: Identification of potential therapeutic drugs for huntington's disease using Caenorhabditis elegans. PLoS One 2007, 2(6):e504.

74. Pulak $R$ : Techniques for analysis, sorting, and dispensing of $C$. elegans on the COPAS flow-sorting system. Methods Mol Biol 2006, 35 I:275-86.

75. Burns AR, Kwok TC, Howard A, Houston E, Johanson K, Chan A, Cutler SR, McCourt P, Roy PJ: High-throughput screening of small molecules for bioactivity and target identification in Caenorhabditis elegans. Nat Protoc 2006, I:1906-14.

76. Kwok TC, Ricker N, Fraser R, Chan AW, Burns A, Stanley EF, McCourt P, Cutler SR, Roy PJ: A small-molecule screen in C. elegans yields a new calcium channel antagonist. Nature 2006, 441:91-5.

77. Boyd WA, Smith MV, Kissling GE, Freedman JH: Medium- and highthroughput screening of neurotoxicants using $C$. elegans. Neurotoxicol Teratol 2009 in press.

78. Yanik MF, Cinar H, Cinar HN, Chisholm AD, Jin Y, Ben-Yakar A: Neurosurgery: functional regeneration after laser axotomy. Nature 2004, 432:822.

79. Rohde CB, Zeng F, Gonzalez-Rubio R, Angel M, Yanik MF: Microfluidic system for on-chip high-throughput whole-animal sorting and screening at subcellular resolution. Proc Natl Acad Sci USA 2007, 104:1389|-5.

80. Chung K, Crane MM, Lu H: Automated on-chip rapid microscopy, phenotyping and sorting of $C$. elegans. Nat Methods 2008, 5:637-43.

81. Ben-Yakar A, Bourgeois F: Ultrafast laser nanosurgery in microfluidics for genome-wide screenings. Curr Opin Biotechnol 2009, 20:100-5.

82. Brignull HR, Moore FE, Tang SJ, Morimoto RI: Polyglutamine proteins at the pathogenic threshold display neuron-specific aggregation in a pan-neuronal Caenorhabditis elegans model. J Neurosci 2006, 26:7597-606.

\section{Publish with Bio Med Central and every scientist can read your work free of charge}

"BioMed Central will be the most significant development for disseminating the results of biomedical research in our lifetime. "

Sir Paul Nurse, Cancer Research UK

Your research papers will be:

- available free of charge to the entire biomedical community

- peer reviewed and published immediately upon acceptance

- cited in PubMed and archived on PubMed Central

- yours - you keep the copyright

Submit your manuscript here:

http://www.biomedcentral.com/info/publishing_adv.asp 